\title{
Funktionen von Modalitätsinterdependenzen in professionellen Interaktionen
}

\author{
Anna Valentine Ullrich, Eva-Maria Jakobs (Aachen)
}

\begin{abstract}
This paper deals with multimodal interaction in professional settings. The question is: How do people use communicative resources to create and pass on knowledge cooperatively? The focus lies on functions of the interplay of multimodal communicative resources. The analyses presented are based on a case study: in a company, industrial process modelers interview employees about workflows, then develop a preliminary process model of the production process and afterwards a computer-generated final process model. In these situations, the participants combine complex resources such as speaking, writing, and visualizing (so-called modality interdependencies, MID). The article proposes a model for describing and analyzing the forms and functions of modality interdependencies.
\end{abstract}

\section{$1 \quad$ Einleitung}

Der Beitrag geht der Frage nach, wie Menschen in professionellen Zusammenhängen kommunikative Ressourcen (hier: Sprechen, Schreiben, Visualisieren ${ }^{1}$ ) nutzen, um kooperativ Wissen zu erzeugen und weiterzugeben. Der Fokus richtet sich dabei auf Funktionen des Zusammenspiels verschiedener kommunikativer Ressourcen. Das simultane oder versetzte Nutzen dieser Ressourcen wird als Modalitätsinterdependenz bezeichnet.

Die Betrachtung von MID-Funktionen stützt sich auf die Analyse eines Beispiels, das in einem Sachgüter produzierenden Unternehmen aufgenommen wurde. Im Fallbeispiel geht es um die Erhebung und Darstellung eines Produktionsablaufes des Unternehmens. Für Zwecke der Überprüfung und Optimierung erheben (d. h. erfragen) Prozessmodellierer (Ingenieure) den Ist-Zustand eines Produktionsablaufs, genauer: wie ein bestimmtes Produkt hergestellt wird (Arbeitsschritte, Akteure, Aufgaben, mögliche Störmomente usw.). Sie machen sich Notizen und stellen die erhobenen Informationen grafisch-symbolisch dar. Die interaktionsbegleitend entstehenden handschriftlichen Notizen dienen situationsübergreifend in nachfolgenden Interaktionssituationen als Arbeits- und Wissensgrundlage für das Zusammenführen von Teilinformationen (vorläufige Modellierung). Die Teilinformationen werden final am Computer in ein digitales Prozessmodell überführt. Die grafisch-symbolischen Darstellungen orientieren sich an den Vorgaben einer ingenieurswissenschaftlichen Notationssprache für Zwecke der Prozessmodellierung.

\footnotetext{
${ }^{1}$ Hier synonym verwendet mit grafisch-symbolisch Darstellen. Zum Begriff der grafisch-symbolischen Darstellung(sformen) in der Wirtschaftskommunikation vgl. Brünner (2000: 235f.).
} 
Das Fallbeispiel umfasst damit drei Interaktionstypen - Interview als Mittel industrieller Prozesserhebung, vorläufige Prozessmodellierung durch externe Experten sowie Übergabe an Dritte und finale Prozessmodellierung, die interaktionslinguistisch über Handlungsschemata beschrieben werden können.

Der Beitrag geht davon aus, dass Modalitätsinterdependenzen als wesentlicher Bestandteil vieler professioneller Interaktionen (vgl. Ullrich/Reimer/Jakobs 2017: 52) funktional bedeutsam sind. Die Ausgangsthese lautet: MID sind im Falle ihres Auftretens in professionellen Interaktionen aufgabenbezogen erwartbar und funktional gebunden, d. h. sie tragen zur Bearbeitung professioneller Aufgaben bei.

Interaktionsspezifische Aufgaben und ihre Einbettung in sprachlich-kommunikative Handlungsschemata und -muster werden seit den 1970er Jahren in der Interaktionslinguistik empirisch rekonstruiert. Ihre Betrachtung basiert auf dem Konzept der Interaktionskonstitution. Beispiele sind Analysen von Beratungs-, Verkaufs-, Reklamations- oder Schlichtungsgesprächen (vgl. Kallmeyer/Schütze 1977, Kallmeyer 1985, Spranz-Fogasy 1997, Spiegel/Spranz-Fogasy 2001, Fiehler/Kindt/Schnieders 2002; zu einem etwas anders gearteten Ansatz der kommunikativen Gattungen und Formen in professionellen Interaktionen vgl. Müller 2006). Die in der Literatur rekonstruierten Handlungsschemata und Interaktionsaufgaben erfassen meist gesprochensprachliche Anteile. Bisher wenig untersucht ist das Zusammenspiel kommunikativer Ressourcen bei der Aufgabenbearbeitung (z. B. Schreibaktivitäten in Befragungssituationen), wie sie für das hier betrachtete Fallbeispiel typisch sind.

Forschungsfragen des Beitrags sind: Wie hängen Modalitätsinterdependenzen funktional mit Handlungen und Aufgaben einer Interaktionssituation zusammen? Welche Funktionen sind im Datenmaterial zentral? Ziel des Beitrags ist, Funktionen von Modalitätsinterdependenzen zu beschreiben und darüber ihre Bedeutung für professionelle Zusammenhänge herauszustellen. Der Beitrag stützt sich auf Forschungsarbeiten des DFG-geförderten Projekts ModiKo².

Kapitel 2.1 beschreibt den Forschungsansatz von ModiKo und das Datenmaterial. Kapitel 2.2 stellt ein in ModiKo entwickeltes Modell vor, das Dimensionen, die wesentlich für die Betrachtung von MID-Formen und Funktionen sind, in einen systematischen Zusammenhang bringt. Kapitel 3.1 beschreibt modellgeleitet den interaktionslinguistischen Zugang zu MID-Funktionen über Handlungsschemata professioneller Interaktionen. Der Beschreibung des methodischen Vorgehens der Analyse von MID-Funktionen (Kap. 3.2) folgen Befunde zu MID-Funktionen. Ausgehend von Zusammenhängen zwischen (Aufgaben der) Handlungschemata und Modalitätsinterdependenzen im Fallbeispiel (Kap. 3.3) werden zentrale MID-Funktionen von Interaktionstypen vorgestellt (Kap. 3.4) und MID bezogen auf ihre Mono- oder Multifunktionalität im Fallbeispiel betrachtet (Kap. 3.5). Kapitel 4 zieht ein Fazit und benennt weiterführende Forschungsfragen.

\footnotetext{
2 Das ModiKo-Projekt „Sprechen - Schreiben - Visualisieren. Formen, Funktionen und Störungen von Modalitätsinterdependenzen und Konzeptgenesen in professionellen Interaktionssituationen“ (GZ: JA 1172/3-1) ist eine Kooperation der Professur Textlinguistik und Technikkommunikation, RWTH Aachen University (Eva-Maria Jakobs) mit dem IDS Mannheim (Reinhard Fiehler und Thomas Schmidt).
} 


\section{Inhaltlicher und methodischer Zugang zu Modalitätsinterdependenzen}

\subsection{Das Projekt ModiKo: Ziele, Daten und Methoden}

Ziel des Projektes ModiKo (2014-2018) ist die Entwicklung von Ansätzen und Methoden, die Formen und Funktionen von Modalitätsinterdependenzen in ihrer Musterhaftigkeit erfassen und beschreiben. Der Ausdruck Modalitätsinterdependenz (MID) ist ein analytisches Konzept zur Untersuchung des professionellen Interaktionsgeschehens. Der Ausdruck MID bezeichnet aus einer pragmatischen Perspektive das Zusammenspiel komplexer kommunikativer Ressourcen (Modalitäten) wie Sprechen, Schreiben und Visualisieren (vgl. Ullrich/Reimer/Jakobs 2017: 52), die situationsabhängig von den Interaktionsbeteiligten zu bestimmten Zwecken kombiniert werden. ${ }^{3}$ Modalitäten stellen ,jeweils spezifische Möglichkeiten zur Verfügung [...], sich in kommunikationsrelevanter Weise auszudrücken, Handlungsziele zu erreichen und soziale Bedeutung zu konstituieren“ (Schmitt 2005: 19). Das Zusammenspiel der Modalitäten ist vergleichbar mit den „Kontaktstellen“ multimodaler Texte, die Stöckl (2006: 27) in den Varianten eines additiven, komplementären, hierarchischen, divergenten oder konfliktiven Verhältnisses von Modalitäten beschreibt. Ausgehend von einer grundlegenden Multimodalität der Kommunikation (vgl. Bucher 2011: 124) stellen Modalitätsinterdependenzen im Fallbeispiel des Beitrags einen Fall des Auftretens von Multimodalität dar. Es ist eine Multimodalität im engeren Sinne (vgl. Fricke 2012: 47), da im Fallbeispiel die Modalitäten Sprechen, Schreiben und Visualisieren mehrere Sinne bedienen, auditive (Ton) und visuelle (Schrift und Zeichnung/Bild).

Die Projektarbeiten basieren auf Daten einer Fallstudie des BMBF-Projektes IMIP „Interdisziplinäre Methoden industrieller Prozessmodellierung“ (2008-2011) zu sprachlichen Anteilen professioneller Methoden der Erhebung von Ist-Zuständen industrieller Produktionsprozesse und ihrer Modellierung (vgl. Jakobs et al. 2011, Jakobs 2011). Ziel des erhobenen Verfahrens ist es, Prozessabläufe in einem Industrieunternehmen so zu erfassen, wie sie ,tatsächlich` ablaufen: So genannte Prozessmodellierer (im Fallbeispiel nur Männer) befragen vor Ort Unternehmensmitarbeiter zu Arbeitsabläufen (Was geschieht an ihrem Arbeitsplatz? Woher kommen die dort bearbeiteten Teile? Wie werden sie bearbeitet? Wohin gehen sie?) (Interaktionstyp 1: Interview als Mittel industrieller Prozesserhebung). Die für die Ist-Analyse zuständigen Personen (im Fallbeispiel zwei Prozessmodellierer) sammeln und diskutieren im Anschluss (Interaktionstyp 2: vorläufige Prozessmodellierung durch externe Experten) das Gehörte und Gesehene, reduzieren es auf wichtige Aussagen, verdauern diese schriftlich oder grafisch-symbolisch und verbinden die Ergebnisse zu einem Prozessmodell des untersuchten Produktionsprozesses (Interaktionstyp 3: Übergabe an Dritte und finale Prozessmodellierung). In den professionellen Interaktionen des Fallbeispiels nutzen die Interaktionsbeteiligten verschiedene kommunikative Ressourcen: Sprechen, Schreiben und Visualisieren. Der analytische Zugang geht von Sprechen als zentraler Aktivität der Interaktionsbeteiligten aus. Schreiben umfasst das Notieren einzelner Stichwörter (Prägung, $M a ß e$ ), Nomen-Verb-Phrasen (Werkzeuge einbauen) und Maßangaben (Zahl-Nomen-Phrasen

\footnotetext{
${ }^{3} \mathrm{Zu}$ Bezügen zwischen Sprechen und Schreiben in professionellen Interaktionen vgl. z. B. Brünner (2000: 237250).
} 
wie 9 Stufen, 3 Stk pro Std.) auf einer Schreibunterlage oder einem Schreibblock. Visualisieren umfasst unterschiedliche Formen grafisch-symbolischen Handelns mit zunehmendem Abstraktionsgrad: individuelles Skizzieren ${ }^{4}$ (ikonisches Zeichnen von Produktionsbestandteilen), grafische Auszeichnungemittel für Hervorhebungen (Umkreisen von Wörtern) und optische bzw. inhaltliche Trennung von Aspekten (Trennlinien auf der Blattfläche) sowie konventionalisierte Elemente der verwendeten Notationssprache (Viereck, Raute, Pfeil) (zu Formen und Funktionen des Visualisierens im Fallbeispiel vgl. Ullrich/Jakobs 2019/in Vorb.). Die Darstellung von Prozesselementen zur Prozessmodellierung erfolgt mit der ingenieurswissenschaftlichen Notationssprache K3; sie enthält grafische Elemente (Vierecke, Verbindungslinien) und sprachliche Elemente (Nomen, nominale Phrasen, Nomen-VerbPhrasen).

Die Gesamtdatenbasis umfasst 548 Minuten Videodaten (.mpeg), 266 Seiten Transkript, (.doc, GAT 2) und 89 Scans interaktionsbegleitend erstellter Notizen (Modelldarstellungen mit schriftlichen und grafisch-symbolischen Anteilen; .jpeg). Die Analyse und Annotation von MID-Formen und -Funktionen wie auch die Entwicklung des Annotationsansatzes stützen sich auf ein aus der Gesamtdatenbasis gebildetes Pilotkorpus (9.045 Token) und einen Goldstandard (33.185 Token; 238 Minuten Videodaten) (siehe Tab. 1 im Anhang).

Die Annotation und Analyse von MID-Formen und -Funktionen erfolgt mit einem iterativ daten- und modellgeleitet entwickelten Mehrebenenannotationsschema, das richtlinienbasiert die semiautomatische Annotation und Analyse von Spontansprache in Transkripten als Teil multimodaler Daten (Videos und Transkripte) in EXMARaLDA (vgl. Schmidt/Wörner 2014) ermöglicht (vgl. Reimer et al. 2017). Der Ansatz nutzt texttechnologische, korpus- und computerlinguistische Ansätze.

ModiKo unterscheidet drei Typen von Daten: Primär-, Sekundär- und Tertiärdokumente. Primärdokumente sind Videodateien der erhobenen Interaktionen sowie Scans interaktionsbegleitend erstellter Notizen und Skizzen. Sekundärdokumente sind multimodale Transkripte der Videodaten, d. h. Transkripte, die angereichert werden durch Informationen zu praktischen und kommunikativen Handlungen der Interaktionsbeteiligten sowie den von ihnen in der Interaktion genutzten Objekten (z. B. der Prozessmodellierer hält dem Interviewten ein Klemmbrett mit einem Notizblatt hin und zeigt darauf). Durch die Mehrebenenannotation der Sekundärdokumente (zur Erfassung und Beschreibung von Modalitätsinterdependenzen) entstehen Tertiärdokumente (vgl. Reimer et al. 2015, Trevisan et al. 2016).

Die Analysen des Fallbeispiels bestätigen die Ausgangsthese, dass MID für das Fallbeispiel interaktional relevant sind - das Korpus weist eine hohe Anzahl von MID auf (vgl. Ullrich/Reimer/Jakobs 2017: 53). Ihre Anzahl und Form variieren abhängig vom

\footnotetext{
${ }^{4}$ Bisweilen liegt im Datenmaterial auch virtuelles Skizzieren der Interaktionsbeteiligten vor - virtuelle, gestische Inskriptionen in der Luft, bevor materielle Inskriptionen auf dem Papier erfolgen (zu virtuellen Inskriptionen vgl. Pitsch/Krafft 2010: 198). Mittelberg, Schmitz und Groninger (2017: 63) beschreiben solche Gesten als „Manifestationen im Sinne eines ,exbodied mind““. Das Videomaterial der Fallstudie erlaubt keine durchgängige Analyse dieser Phänomene (zu Gesten in der Interaktion vgl. z. B. Müller 2010).
} 
Interaktionstyp. Die meisten MID finden sich in Interaktionstyp 2 (vorläufige Modellierung), der sich durch eine Vielzahl kooperativer Schreib- und Visualisierungshandlungen auszeichnet.

\subsection{Formen von Modalitätsinterdependenzen}

Für die formale Beschreibung von MID-Formen und den Zugang zu MID-Funktionen wurde ein Modell entwickelt, das die Sicht auf den Gegenstand leitet. Die Entwicklung erfolgte theoriebasiert an Ankerbeispielen (vgl. Ullrich/Jakobs/Eraßme 2016), das Modell wurde schrittweise verfeinert (vgl. Ullrich/Reimer/Jakobs 2017). Die Endversion beschreibt Dimensonen, die für MID-Formen und -Funktionen aus Makro- und Mikroperspektive relevant sind (Abb. 1). Die Makroperspektive berücksichtigt den allgemeinen Situationstyp (im Fallbeispiel die professionelle Situation der Prozesserhebung und -modellierung in einem Unternehmen), in den die oben genannten Interaktionstypen und die sie beschreibenden Handlungsschemata eingebettet sind. Letztere umfassen Rollen und Aufgaben der Interaktionsbeteiligten (vgl. Kap. 3.1). Die Mikroperspektive verschränkt die zeitliche Betrachtung (horizontal) von Interaktionsverläufen mit interaktionalen Betrachtungsdimensionen (vertikal), die sich im Verlauf der Analyse datenbasiert als notwendig und sinnvoll erwiesen.

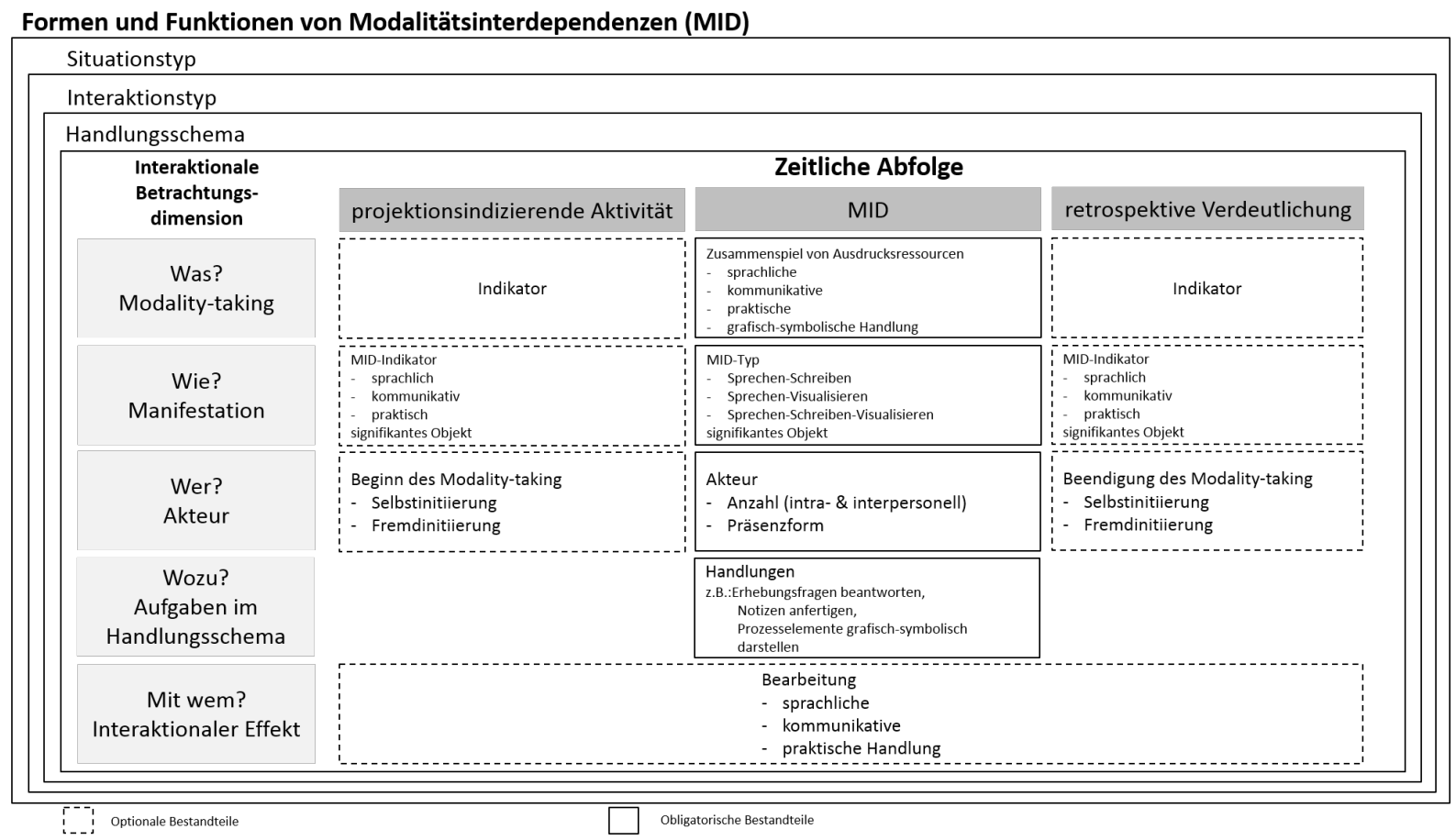

Abbildung 1: Modell zur Beschreibung der Formen und Funktionen von Modalitätsinterdependenzen

Die Sicht auf MID-Formen und -Funktionen orientiert sich an fünf Leitfragen: Was geschieht bei einer MID (Modality-taking)? Wie manifestiert sich eine MID in der konkreten Situation (Manifestation)? Wer ist an einer MID beteiligt (Akteur)? Wozu dienen MID (Aufgaben im Handlungsschema)? Wer ist nicht an einer MID beteiligt, aber Teilnehmer der Interaktionssituation, in der die MID auftritt (interaktionaler Effekt)? Im Folgenden werden die Betrachtungsdimensionen kurz erläutert.

- Modality-taking meint die aktive Zuwahl einer Ausdrucksressource durch einen Interaktionsbeteiligten, die zu einer MID führt (z. B. Visualisieren zum Sprechen). Im 
Fallbeispiel bedingt die professionelle Interaktionssituation Verbalität als primäre Modalität, die anforderungsbezogen durch weitere Modalitäten ergänzt wird und MID nach sich zieht. Bestimmte Handlungen können analytisch auf eine MID hinweisen und damit als Indikator für MID fungieren (z. B. metakommunikative Äußerungen, dass geschrieben oder gezeichnet wird).

- Manifestation erfasst das spezifische Auftreten von MID-Indikatoren und -Konstellationen. Indikatoren können einer MID vorgelagert (projektionsindizierende Aktivität) oder nachgelagert (retrospektive Verdeutlichung) sein. Das Fallbeispiel weist drei Arten von MID-Indikatoren auf: sprachliche Handlungen (z. B. MID anzeigende Substantive wie Fallunterscheidung, ein Begriff aus der K3-Notationssprache), kommunikative Handlungen (z. B. Zeigegesten auf Notizen) und praktische Handlungen (z. B. das Aufkleben von Klebezetteln auf einen Schreibblock als Auftakt zum Schreiben). Im Datenmaterial zeigt sich ein Zusammenhang zwischen MID-Indikatoren und Interaktionstyp: z. B. treten in der vorläufigen Modellierung (Interaktionstyp 2) besonders zwei sprachliche MID-Indikatoren auf - die Verben schreiben und malen; in der finalen Modellierung (Interaktionstyp 3) ist modellieren relevant (vgl. Ullrich/Reimer/Jakobs 2017: 72-73).

In fast allen Interaktionen liegen spezielle MID-Konstellationen vor (vgl. Tab. 2 im Anhang). Es gibt drei Typen: Sprechen-Schreiben, Sprechen-Visualisieren und SprechenSchreiben-Visualisieren. Der MID-Typ Sprechen-Schreiben tritt mit Abstand am häufigsten auf (besonders in Interaktionstyp 1 und 2). Die meisten MID finden sich in Interaktionstyp 2, in dem die beiden Prozessmodellierer schriftlich und grafisch-symbolisch den Produktionsablauf im Unternehmen modellieren. In Interaktionstyp 3 ist der MID-Typ Sprechen-Visualisieren zentral. Das Auftreten von MID ist verbunden mit der Nutzung von Objekten, die in der Interaktion signifikant werden (z. B. Schreibblock und Klebezettel als Arbeitsmittel der Prozessmodellierer oder punktuell auch Produktionsbestandteile als Anschauungs- und Klärungsobjekte) (zu signifikanten Objekten vgl. Schmitt 2013: 40f.).

- Akteur erfasst die Akteure einer MID - ihre Anzahl (intra- und interpersonelle MID), ihre Präsenzform (Stehen, Sitzen oder Bewegen im Raum) sowie akteursbezogene metakommunikative Ankündigungen von MID (Selbst- und Fremdinitiierung). Selbstinitiierungen sind explizite mündliche Ankündigungen eines Modality-takings (z. B. das muss ich mir mal grad aufschreiben); bei Fremdinitiierung fordert ein Interaktionsbeteiligter einen anderen auf, eine Ausdrucksressource zu nutzen (z. B. schreiben sie es mal rein). In Interaktionstyp 1 (Befragung von Mitarbeitern) treten z. B. Selbstinitiierungen häufig im Zusammenhang mit frühen Schreibaktivitäten des Prozessmodellierers auf (vgl. Ullrich/Jakobs/Eraßme 2016: 77). Aus analytischer Perspektive mit dem Schwerpunkt auf MID lassen sich Selbst- und Fremdinitiierung als Hinweis auf Schreib- und Visualisierungshandlungen nutzen.

Akteursbezogen sind intra- und interpersonelle MID zu unterscheiden. Intrapersonelle MID entstehen, wenn ein Interaktionsbeteiligter z. B. spricht und schreibt. Interpersonelle MID entstehen, wenn mindestens zwei Interaktionsbeteiligte in sequentiellen und/oder simultanen Sprech-, Schreib- und Visualisierungshandlungen Inhalte kooperativ aushandeln. Die Analysen zeigen einen Zusammenhang zum Interaktionstyp: In den stark 
kooperativen Interaktionstypen 1 und 2 dominieren interpersonelle MID, in Interaktionstyp 3 dagegen intrapersonelle MID.

- Aufgaben im Handlungsschema: Die Dimension erfasst Handlungen der Interaktionsbeteiligten zur Bearbeitung interaktionaler Aufgaben (z. B. Prozesselemente visualisieren).

- Interaktionaler Effekt im Kontext von MID erfasst, wie die Interaktionsbeteiligten mit dem Modality-taking ihrer Gesprächspartner umgehen (z. B. eine Kooperativität anzeigende Side-by-Side-Stellung des Befragten neben dem sich Notizen machenden Interviewer und Blickwendung auf dessen Schreibblock).

\section{Funktionen von Modalitätsinterdependenzen}

\subsection{Handungsschematische Betrachtung}

Ausgangspunkt der funktionalen Untersuchung von MID im Datenmaterial ist ihre handlungsschematische Einordnung. Jakobs et al. (2011: 229f.) beschreiben das im Fallbeispiel betrachtete Verfahren der industriellen Prozesserhebung top-down in einem mehrstufigen Ebenenmodell: Auf der obersten Ebene wird stark abstrahiert der Gesamthandlungszusammenhang der Methodik der industriellen Prozessmodellierung als Abfolge funktionaler Phasen (nach Allweyer 2009: 97) beschrieben (Planung, Ist-Zustand, Soll-Zustand, Implementierung Soll-Konzept, kontinuierliches Prozessmanagement). ModiKo fokussiert die Phase „Erfassung des Ist-Zustandes eines Produktionsprozesses“. Die zweite Ebene bildet die Substruktur der Phasen ab, hier die Substruktur der Phase „Erfassung des IstZustandes“. Auf der dritten und letzten Ebene werden fallbeispielbezogen dazugehörige Interaktionstypen und Handlungsschemata betrachtet.

Der Betrachtung von Handlungsschemata liegt ein theoretisches Verständnis von Kommunikation zugrunde, dass $\mathrm{u}$. a. die interaktive Hervorbringung und Zweckgebundenheit von Kommunikation, ihre Musterhaftigkeit sowie die Notwendigkeit von Perspektivenübernahmen in der Interaktion betont (vgl. Fiehler/Kindt/Schnieders 2002: 131f.).

Grundlegend für die Rekonstruktion eines Handlungsschemas ist die Frage, was für einen bestimmten Gesprächstyp konstitutiv ist. Ziel der Beschreibung von Schemata ist primär die Analyse auftretender kommunikativer Probleme und weitergehend die Entwicklung von Lösungsansätzen für kommunikative Probleme (vgl. Habscheid et al. 2015: 400-406). In ModiKo wird die analytische Rekonstruktion der Handlungsschemata genutzt, um Aussagen über den Zusammenhang des Auftretens von Modalitätsinterdependenzen und Aufgaben im Interaktionstyp zu machen.

Jakobs et al. (2011) beschreiben die Handlungsschemata der Interaktionstypen des Fallbeispiels über kommunikative Aufgaben, ihnen zuzuordnende Handlungen sowie Rollen der Interaktionsbeteiligten. Ihre Rekonstruktion stützt sich im Wesentlichen auf Kallmeyer (1985), Fiehler, Kindt und Schnieders (2002) sowie Fiehler (2009), die Gespräche als komplexe Hierarchien kommunikativer Aufgaben verstehen. Diese Hierarchien enthalten elementare Aufgaben mündlicher Interaktion sowie kooperativ zu bearbeitende, interaktionstypspezifische Aufgaben, ihnen zuzuordnende sprachliche Handlungen sowie Rollen der Interaktionsbeteiligten. 
Die Handlungsschemata der drei Interaktionstypen des Fallbeispiels lassen sich - verkürzt wie folgt beschreiben (vgl. Jakobs et al. 2011: 231-232):

- Das Handlungsschema 1 „Interview (als Mittel der industriellen Prozessmodellierung)“ umfasst die Rollen Interviewer (Prozessmodellierer) und Interviewter (Mitarbeiter des Unternehmens). Aufgabenabhängig können weitere Rollen hinzukommen oder Akteure zwischen Rollen wechseln (z. B. fordert der Prozessmodellierer den Befragten manchmal auf, bestimmte Fertigungsteile oder Produktionsabläufe zu skizzieren). Kooperativ zu bearbeitende kommunikative Aufgaben sind: Aufgaben der Interaktionseröffnung (Formen der Kontaktaufnahme wie Begrüßen des Interviewpartners, Selbstvorstellung, Vorstellen der Ziele der Erhebung, Verdeutlichen des zugrunde liegenden Interaktionstyps u. a.), der Informationserhebung (Informationen erfragen zu praktischen und kommunikativen Tätigkeiten, Ablauforganisation, Verfahren, Materialien, Werkzeugen, Schwachstellen und Verbesserungsmöglichkeiten des zu erhebenden Prozesses; Erhebungsfragen beantworten, Prozessaspekte beschreiben und erläutern; Verständnis sichern durch Aktivitäten wie Rückmeldesignal, Reformulierung, Nachfrage u. a.), der Informationsverdauerung (Notizen anfertigen, grafisch-symbolisches Darstellen von Inhalten u. a.) und der Interaktionsbeendigung (Danksagung, Verabschiedung u. a.).

- Das Handlungsschema 2 „Vorläufige Prozessmodellierung durch externe Experten“ umfasst die Rollen Rekonstruierender und Prozessmodellierer, zwischen denen die Beteiligten aufgabenbezogen wechseln können. Kooperativ zu bearbeitende kommunikative Aufgaben beziehen sich auf die Arbeitsorganisation (wie Vorgehen, Rollen, Aufgaben und Nutzung von Arbeitsmaterialien klären), die Informationsrekonstruktion (erhobene Informationen (anhand von Beobachtungen, Notizen, erinnerter Interviewaussagen) rekonstruieren; Wissenslücken, Informationsbedarf u. a. identifizieren) und die Prozessmodellierung (Prozesselemente und -relationen kooperativ identifizieren, reduzieren, abstrahieren, anordnen und benennen, Notationselemente auswählen, Prozesselemente und Relationen zwischen Elementen grafisch-symbolisch und schriftlich notieren u. a.).

- Das Handlungsschema 3 ,Übergabe an Dritte und finale Prozessmodellierung durch externe Experten" umfasst die Rollen Informant und Prozessmodellierer sowie den möglichen Wechsel zwischen den Rollen. Kooperativ zu bearbeitende Aufgaben sind: Aufgaben der Informationsübergabe (Gegenstand festlegen, etwa durch Benennen des Prozesses; Prozessausschnitte anhand von Skizzen und Notizen der Prozesserhebung und vorläufigen Prozessmodellierung beschreiben, Angabe zusätzlicher Informationen, Benennen zusätzlich zu modellierender Elemente u. a.), der Erstellung des Prozessmodells (das vorläufige Prozessmodell in Softwaretools eingeben, ergänzen u. a.), der Korrektur des Prozessmodells (Notationselemente umbenennen, zusätzliche Notationselemente einfügen u. a.) sowie der Ratifizierung (Freigabe des Modells u. a.).

Die Ziele und Anforderungen der Methodik industrieller Prozessmodellierung leiten das Handeln der Interaktionsbeteiligten: Sie geben eine zeitliche und inhaltliche Abfolge von Interaktionstypen vor, bestimmte Interaktionsformate (z. B. Interview) mit speziellen Aufgaben, die zu Modalitätsinterdependenzen führen, wie das Erfragen, Dokumentieren und grafisch-symbolische Darstellen von Sachverhalten (Visualisieren). Visualisieren gilt in der 
Literatur zur industriellen Prozessmodellierung als Mittel der Objektivierung einer Sachverhaltsdarstellung: „Wer nicht visualisiert, setzt sich der Willkür aus“, schreiben Best und Weth (2007: 69) in ihrem Praxisleitfaden zur Geschäftsprozessoptimierung. Visualisieren bedeutet in diesem Zusammenhang eine zeichnerische, skizzenhafte Darstellung (seitens der Prozessmodellierer) von Bestandteilen oder Abläufen des Produktionsprozesses zur Klärung und Ratifizierung bestimmter Sachverhalte durch die befragten Unternehmensmitarbeiter. Damit kann auch eine kooperative Zusammenarbeit zwischen Prozessmodellierer und Befragten in der Interviewsituation ermöglicht werden. Im weiteren Sinne bezeichnet Visualisieren die abstrakt-formalisierte Darstellung des Produktionsprozesses in der Notationssprache K3. In der ingenieurwissenschaftlichen Perspektive sind Visualisierungen wesentlich für das Erfassen von Zusammenhängen (nach dem Motto „Das Bild ist die Sprache des Ingenieurs.“)

\subsection{Methodisches Vorgehen}

Die Analyse von MID-Funktionen orientiert sich fallbeispielbezogen an den durch die Interaktionsbeteiligten zu bearbeitenden Aufgaben und Handlungen ihrer Bearbeitung. ${ }^{5}$ Die Ausgangsthese lautet: MID sind funktional an die Umsetzung einer oder mehrerer Aufgaben gebunden und damit inhärenter Bestandteil der kooperativen Arbeitssituation.

Für die Erfassung von Funktionen wird das Mehrebenenannotationsschema erweitert um die Ebene Handlungen. Im Datenmaterial werden Handlungen der Interaktionsbeteiligten annotiert, die zur Bearbeitung der Aufgaben eines Interaktionstyps beitragen (Handlungsschemata 1 bis 3) und eine MID aufweisen, z. B. Prozesselemente grafischsymbolisch darstellen, erhobene Informationen (anhand von Beobachtungen, Notizen, erinnerter Interviewaussagen) rekonstruieren oder Notizen korrigieren. Die Identifizierung der analyserelevanten Datenausschnitte orientiert sich damit an den im Datenmaterial ausgezeichneten MID-Aufkommen (annotiert als MID-Typ, z. B. Sprechen-Schreiben) und der Rekonstruktion der sie einbettenden Handlungen und Aufgaben.

Für jedes Handlungsschema wird im Annotationsschema eine eigene Handlungsebene angelegt. Die einer Handlung zuzuordnende Aufgabe wird mit einem Tag annotiert, das die jeweilige Handlung und Aufgabe über Kürzel-Kombinationen erfasst. Die funktionale Analyse erfolgt nacheinander für jeden Interaktionstyp und die dazugehörigen Daten. Die Annotation von Funktionen erfolgte primär händisch.

Zunächst wurde das Pilotkorpus in Einzelannotationen von Subkorpora von drei Annotatoren annotiert. Anhand der Daten wurden in Workshops Probleme diskutiert und Annotationen validiert. Die Analyse und Annotation der Daten zeigte, dass die in Jakobs et al. (2011) beschriebenen Handlungsschemata Handlungen übersehen, die relevant für das Datenmaterial sind und eine Erweiterung der Handlungsschemata bedingen. Die Erweiterung betrifft zum einen Korrekturhandlungen, die in allen drei Interaktionstypen auftreten, zum anderen Handlungen, die nur in einzelnen Handlungsschemata beobachtbar sind. In Handlungsschema

\footnotetext{
${ }^{5}$ Der Fokus liegt nicht auf interaktionskonstitutiven Aufgaben wie sie etwa Hausendorf (2015: 56f.) zusammenfassend darstellt, sondern auf den spezifischen Aufgaben der professionellen Interaktion.
} 
1 wurde die übergeordnete Handlung des Verständnissicherns durch die untergeordnete akteurbezogene Handlung Selbstklärung ergänzt (z. B. mündliches Aussprechen des zu Schreibenden). In Handlungsschema 2 wurde die Handlung Prozesselemente und -relationen kooperativ identifizieren, reduzieren, abstrahieren usw. durch ratifizierendes Bestätigen ergänzt. Das erweiterte Annotationsschema wurde anhand des Goldstandards erprobt und verifiziert.

\subsection{Zusammenhänge zwischen Handlungsschema und Modalitätsinterdependenzen: Ergebnisse}

Die Analysen des Fallbeispiels zeigen, dass nicht alle Aufgaben der Handlungsschemata mit MID einhergehen, aber alle auftretenden MID mit der Umsetzung einer oder mehrerer Aufgaben der professionellen Interaktion verknüpft sind. Basale kommunikative Aufgaben wie Interaktionseröffnung und -beendigung sind nie mit MID verbunden. Alle interaktionstypspezifischen Aufgaben (z. B. die Informationsverdauerung in Handlungsschema 1) bewältigen die Interaktionsbeteiligten dagegen systematisch, indem sie kommunikative Ressourcen kombinieren. Ausnahmen sind die Aufgaben Erstellung des Prozessmodells und Ratifizierung (Handlungsschema 3). Sie sind im Datenausschnitt (Goldstandard) nicht vertreten.

Die kombinierte Nutzung von Modalitäten konstituiert die Arbeitssituation in den drei Interaktionstypen und ermöglicht den Interaktionsbeteiligten in unterschiedlichen Varianten, die komplexen interaktiven Aufgaben zu bewältigen. Neben die Kernaktivität Verbalität tritt phasenweise Schreiben sowie Visualisieren (mal als grafisch-symbolischer Anteil der Notationssprache, mal als Mittel der Verdeutlichung). Ziel der professionellen Aufgaben im Fallbeispiel ist die digitale Modellierung des Ist-Zustandes des Produktionsprozesses in der Notationssprache K3. Auf dem Weg zu diesem Ziel verändert sich der Einsatz von Modalitäten und ihr Verhältnis zueinander. Während im Interview für die Prozessmodellierer Befragen und Notizenmachen im Vordergrund steht, werden in der vorläufigen Modellierung Notizen durchgesehen und besprochen, gemeinsam neue Darstellungen entwickelt und diese verschriftlicht. In der finalen Modellierung werden die Teildarstellungen erläutert, am PC in einem Gesamtmodell zusammengeführt, das Ergebnis ausgedruckt und anhand der Ausdrucke korrigiert. D. h. im Verlauf des Modellierungsprozesses werden - anforderungs- und aufgabenbezogen - spezifische Potenziale der Modalitäten und ihres Zusammenspiels relevant.

Die interaktive Aufgaben- bzw. Themenbearbeitung folgt keiner linearen Struktur (ähnliche Beobachtungen finden sich in der Literatur zur Handlungsschemaanalyse, vgl. etwa Spiegel/Spranz-Fogasy 2001: 244). Die Interaktionsbeteiligten springen in der Bearbeitung anstehender Aufgaben hin und her, so z. B. im Interview zwischen Informationserhebung und -verdauerung. Die Verteilung der Rollen zwischen den Interaktionsbeteiligten ist situationsbezogen variabel. Phasenweise werden sie anders als konventionell erwartbar besetzt: In der Interviewsituation (Handlungsschema 1) fordert z. B. ein Prozessmodellierer mehrmals die Befragten auf, sich an der Aufgabe der Darstellung und Verdauerung des betreffenden Prozessabschnitts zu beteiligen und reicht ihnen Blatt und Stift weiter. 
Abbildung 2 (siehe nächste Seite) veranschaulicht exemplarisch für Handlungsschema 1 von links nach rechts das Handlungsschema, das Zusammenspiel von Aufgaben des Handlungsschemas, Handlungen ihrer Bearbeitung (Kontaktaufnahme, Informationen erfragen, Verständnis sichern usw.), die dabei genutzten Modalitäten ( $\mathrm{Sp}=\mathrm{Sprechen}$, $\mathrm{Sc}=$ Schreiben, $\mathrm{V}=$ Visualisieren) sowie - rechts außen - die für Schreib- und Visualisierungshandlungen genutzten Objekte (z. B. Ikons für Objekte wie Stift und Klebezettel). Kommunikate Aufgaben, die nicht mit MID einhergehen, sind oval umrandet, interaktionstypspezifische Aufgaben mit MID sind rechteckig gerahmt.

Interaktionstypbezogen werden Aufgaben durch (mehrere) typische Handlungen erfüllt (z. B. Informationsverdauerung durch Inhalte grafisch-symbolisch darstellen, Notizen anfertigen und Notizen korrigieren). Interessant ist, dass manche Handlungen nur mit bestimmten, andere dagegen mit unterschiedlichen kommunikativen Ressourcen ausgeführt werden. So erfolgt das Verständnissichern in Handlungsschema 1 modal mündlich oder schriftlich und/oder grafischsymbolisch, ebenso das Beantworten von Erhebungsfragen. Auch bei Handlungsschema 2 finden sich Handlungen, die modal , unspezifisch` oder offen sind (z. B. das Rekonstruieren erhobener Informationen - mal mündlich, mal schriftlich). Ein Erklärungsansatz wäre, dass bei der Wahl der Modalitäten Faktoren wie situationale Anforderungen, berufsbedingte Prägungen (z. B. grafische Darstellungskonventionen in der ingenieurswissenschaftlichen Ausbildung der Prozessmodellierer bzw. die generelle Wertschätzung visueller Darstellungsformen in dieser Disziplin) oder auch persönliche Affinitäten eine Rolle spielen.

Interaktionstypbezogen werden Aufgaben durch (mehrere) typische Handlungen erfüllt (z. B. Informationsverdauerung durch Inhalte grafisch-symbolisch darstellen, Notizen anfertigen und Notizen korrigieren). Interessant ist, dass manche Handlungen nur mit bestimmten, andere dagegen mit unterschiedlichen kommunikativen Ressourcen ausgeführt werden. So erfolgt das Verständnissichern in Handlungsschema 1 modal mündlich oder schriftlich und/oder grafischsymbolisch, ebenso das Beantworten von Erhebungsfragen. Auch bei Handlungsschema 2 finden sich Handlungen, die modal ,unspezifisch“ oder offen sind (z. B. das Rekonstruieren erhobener Informationen - mal mündlich, mal schriftlich). Ein Erklärungsansatz wäre, dass bei der Wahl der Modalitäten Faktoren wie situationale Anforderungen, berufsbedingte Prägungen (z. B. grafische Darstellungskonventionen in der ingenieurswissenschaftlichen Ausbildung der Prozessmodellierer bzw. die generelle Wertschätzung visueller Darstellungsformen in dieser Disziplin) oder auch persönliche Affinitäten eine Rolle spielen. 
Interaktionstyp 1

Handlungsschema

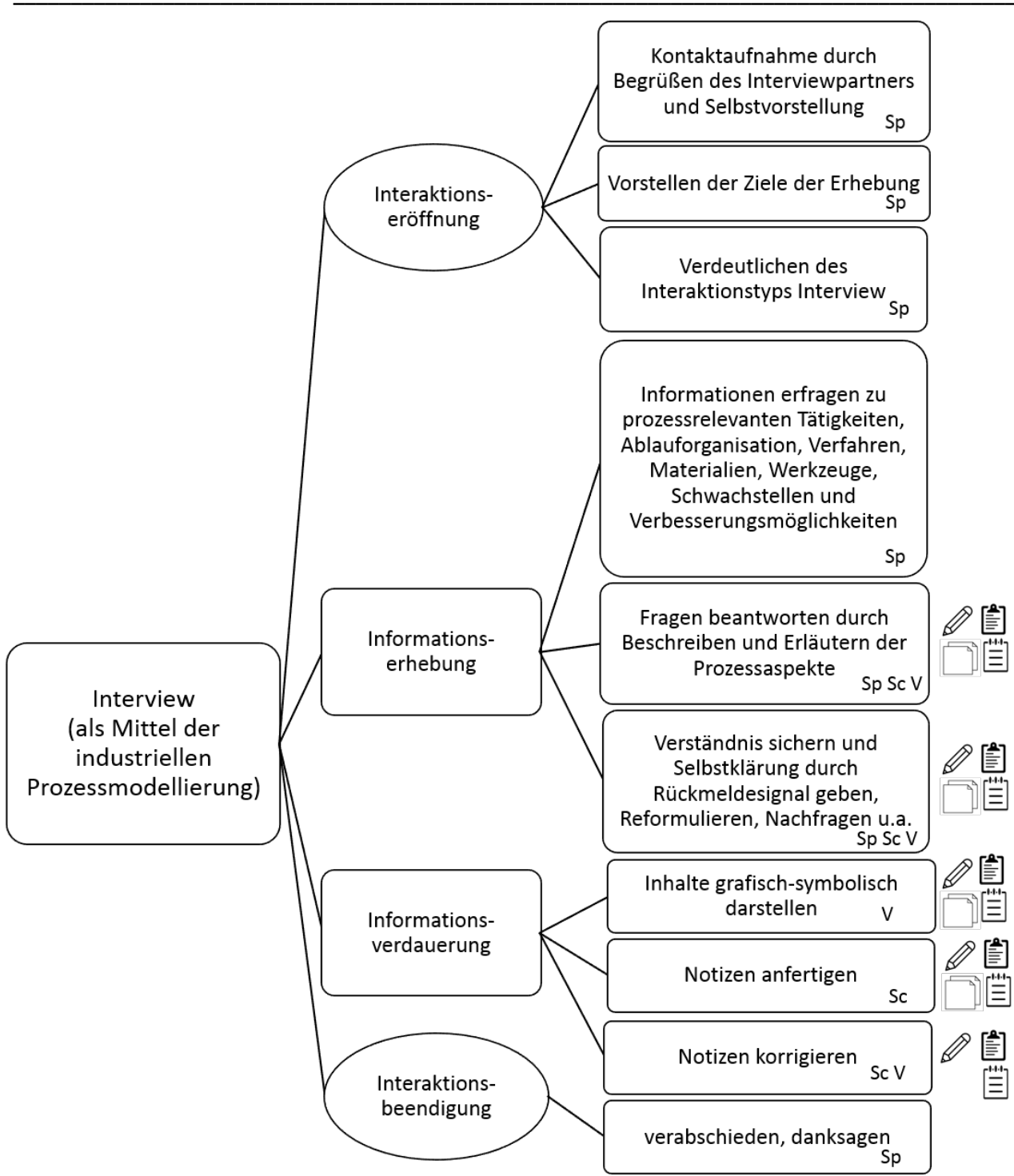

Potentiell beteiligte Modalitäten: $\mathrm{Sp}=$ Sprechen; $\mathrm{Sc}=$ Schreiben; $\mathrm{V}=$ Visualisieren Kasten: Aufgabe mit MID verknüpft; oval: Aufgabe nicht mit MID verknüpft

Objekte: Stift Klebezettel [?] Schreibblock $\left[\begin{array}{l}{[-1+1} \\ \Xi\end{array}\right.$

Abbildung 2: Handlungsschema zu Interaktionstyp 1 des Fallbeispiels

Schreiben und Visualisieren setzen voraus, dass dafür situativ Objekte genutzt werden (z. B. Stift, Schreibblock, Computer). Mit diesen Objekten entstehen im Fallbeispiel interaktionsbegleitend und phasenweise in kooperativen Schreib- und Visualisierungshandlungen Notizen mit verbalen und grafisch-symbolischen Anteilen. Diese Notizen sind interaktionale Produkte der Aufgabenbearbeitung, aber auch Instrumente, die es erlauben, Wissen zum Produktionsprozess in der Situation festzuhalten, darzustellen, zu verdauern und situationsübergreifend im Modellierungsprozess für verschiedene Zwecke zur Verfügung zu stellen. Die entstehenden Kommunikate sind, wie Pitsch (2007: 67) es für Unterrichtskommunikationen mit Tafelskizzen und Schülermitschriften beschreibt, als 
„Ressourcen im Stabilisierungsprozess von erarbeiteten Inhalten“ zu verstehen. Die Notizen des Fallbeispiels, die in den Interviews, den vorläufigen und finalen Modellierungssituationen entstehen, wurden bislang im Projekt nicht systematisch untersucht - sie sind Gegenstand von Folgeuntersuchungen (vgl. Ullrich/Jakobs 2019/in Vorb.).

\subsection{Funktionen von Modalitätsinterdependenzen pro Interaktionstyp}

Die Auswertung zu MID-Funktionen zeigte, dass im betrachteten Datenmaterial MID in zwei Varianten auftreten: 1) MID treten innerhalb einer Handlung auf (z. B. Handlungsschema 1: Aufgabe Informationserhebung, Handlung Fragen beantworten durch Beschreiben und Erläutern der Prozessaspekte kann mündlich und begleitend schriftlich erfolgen; vgl. Abb. 2). ${ }^{6}$ 2) MID treten in der Kombinatorik von Handlungen und Aufgaben auf (z. B. Handlungsschema 1: Aufgaben Infomationserhebung und -verdauerung, Handlungen mündliches Informationen erfragen und schriftliches Notizen anfertigen). In beiden Varianten sind MID funktional.

Abhängig vom Interaktionstyp sind MID unterschiedlich funktional. Die wichtigsten Funktionen von MID sind Informationssicherung (Interaktionstyp 1), interaktive Rekonstruktion und Formalisierung von Informationen (Interaktionstyp 2) sowie Erläuterung und Revision dokumentierter Sachverhalte (Interaktionstyp 3). MID-Typen können je nach Interaktionstyp verschiedene Funktionen haben: In der vorläufigen Modellierung (Interaktionstyp 2) ist der häufig auftretende MID-Typ Sprechen-Schreiben interpersonell annähernd gleich oft mit den Aufgaben Informationsrekonstruktion und Prozessmodellierung (Handlungen Prozesselemente kooperativ identifizieren und schriftlich notieren) oder nur mit der Prozessmodellierung verknüpft. In der finalen Modellierung ist der intrapersonelle MIDTyp Sprechen-Visualisieren typischerweise mit der Aufgabe der Informationsübergabe und den Handlungen zusätzliche Informationen geben oder Prozessausschnitte anhand von Skizzen und Notizen der Prozesserhebung und vorläufigen Modellierung beschreiben verbunden. Hier verdeutlicht ein Prozessmodellierer einem anderen, der nicht an der Erhebung beteiligt war, verbal und in Zeichnungen Elemente des Produktionsprozesses im untersuchten Unternehmen. Im Folgenden werden Ergebnisse zu MID-Funktionen bezogen auf Interaktionstypen dargestellt.

Interaktionstyp 1: In der Interviewsituation sind MID funktional hauptsächlich für die Informationssicherung bedeutsam. Die meisten MID (92\%) lassen sich Handlungen zuordnen, die sich auf die Erfüllung der Aufgaben Informationserhebung und -verdauerung beziehen (z. B. Verständnis sichern und Notizen anfertigen). Nur in geringem Maße sind MID mit nur einer Aufgabe verbunden (Aufgabe der Informationsverdauerung in 7\% der MID-Fälle). Die Handlung des Notizenanfertigens ist, in unterschiedlichen Kombinationen mit Sprechhandlungen wie Erhebungsfragen beantworten oder Informationen erfragen, interaktionstypprägend ( $97 \%$ aller MID-Fälle).? Folglich sind hier auch typische Funktionen

\footnotetext{
${ }^{6}$ Handlungen wie Fragen beantworten, die mündlich, schriftlich und/oder visuell erfolgen, machen MID erwartbar. Es tritt auch der Fall auf, dass nur eine Modalität eingesetzt wird und erst in der Kombination mit anderen Handlungen MID entstehen.

${ }^{7}$ In 7\% der Fälle werden Inhalte auch grafisch-symbolisch dargestellt, meist um Prozessbestandteile und -abläufe im Austausch mit den Befragten zu klären und zu veranschaulichen.
} 
des Schreibens und der Schrift relevant wie Externalisierung und Verdauerung von Gedachtem. In den Interviewsituationen geht es allerdings nicht um ein privates Notizenanfertigen, sondern um ein ,öffentliches', mit den Interviewpartnern geteiltes und phasenweise gemeinsames Notieren (vgl. Brünner 2000). Folglich ist die Kombination von Schreiben und Verbalität zentral, wie es z. B. Mondada und Svinhufvud (2016) in einer konversationsanalytischen Studie beschreiben. Sie sprechen von der Sozialität des Schreibens in der Interaktion als multimodale soziale Praxis. Die Sozialität des Schreibens zeigt sich im Datenmaterial z. B. darin, dass die Handlung des Verständnissicherns (Aufgabe Informationserhebung) im interaktiven Miteinander, d. h. bei interpersonellen MID mit mindestens zwei Interaktionsbeteiligten, relevant ist (39\% aller MID-Fälle). Im Gespräch mit den Unternehmensmitarbeitern versuchen die Prozessmodellierer, die Sicht der Befragten auf ihre Aufgaben, einzelne Arbeitsschritte und den gesamten Produktionsablauf zu erfassen.

Interaktionstyp 2: In der vorläufigen Modellierung sind die Funktionen Rekonstruktion und Formalisierung (Prozessmodellierung) erhobener Sachverhalte primär. Besonders häufig wird die Aufgabe der Prozessmodellierung mit MID bearbeitet (77\% aller MID-Fälle), in 66\% aller Fälle in Kombination mit der Aufgabe der Informationsrekonstruktion (umgesetzt in unterschiedlichen Handlungskombinationen). MID im Zusammenhang mit der Aufgabe der Arbeitsorganisation der Prozessmodellierer sind wenig vertreten (3\%) (z. B. von Schreiben und Visualisieren begleitete Phasen, in denen die Prozessmodellierer diskutieren, ob und wie sie die erhobenen Informationen in der Notationssprache darstellen sollen).

Zentral in diesem Interaktionstyp sind Phasen der Verständigung über das Gehörte und Gesehene bei der Prozessaufnahme, in denen die Modellierer Prozesselemente und ihre Darstellung in interpersonellen MID vom Typ Sprechen-Schreiben aushandeln. Die Klärung von Sachverhalten ist eine wesentliche Funktion des Schreibens in der Interaktion, die die kooperative Schreibforschung beschreibt (vgl. z. B. Lehnen 2017). Durch die Kombination von intersubjektivem Klären und Materialisierung der (Zwischen-)Ergebnisse auf der Schreibfläche vor den Beteiligten wird eine höhere Exaktheit der Darstellung und die Kondensierung komplexer Zusammenhänge möglich (vgl. Lehnen/Gülich 1997).

Interaktionstyp 3: In der finalen Modellierung dienen MID funktional typischerweise der Erläuterung erfasster Sachverhalte (Informationsübergabe) und der Revision einzelner Elemente des erstellten Prozessmodells. Besonders präsent ist die Informationsübergabe an den Prozessmodellierer, der das Prozessmodell in der Notationssprache K3 erstellt. Hier sind intrapersonelle MID vorherrschend (z. B. visualisiert der übergebende Prozessmodellierer mehrmals seine erläuternden mündlichen Ausführungen.) Die Informationsübergabe wird üblicherweise durch die Handlung zusätzliche Informationen angeben und zu modellierende Elemente benennen umgesetzt (53\% der auftretenden MID).

Die Funktion der Revision, des Korrigierens von Teilen der Prozessdarstellung im Rahmen von MID (schriftlich und grafisch-symbolisch), spielt eine wichtige Rolle: Etwas weniger als die Hälfte der MID (46\%) dienen inhaltlichen Korrekturen von Elementen der K3-Modellierung. 12\% der MID sind auch mit Korrekturen des Layouts der K3-Darstellung verknüpft (z. B. die Begradigung von Verbindungslinien zwischen Aktivitäten im Prozessablauf). Die Korrekturen können daran liegen, dass der an der Erhebung nicht beteiligte Prozessmodellierer, der das K3- 
Gesamtmodell erstellt, anhand der Notizen und der Informationsübergabe den Produktionsprozess nicht in allen Einzelheiten nachvollziehen und darstellen konnte. Möglicherweise spielt auch der zeitliche Abstand zur Prozessaufnahme (3 Monate vorher) eine Rolle, der zu etwas anderen Einschätzungen des Prozesses und damit zu Korrekturen führt.

Die Annahme auf Basis der Pilotkorpusuntersuchung, dass Korrekturhandlungen auch in den Interaktionstypen 1 und 2 relevant sind, konnte sich nicht bestätigen. In den Interviewsituationen sind nur vereinzelt Korrekturhandlungen festzustellen (2\%), ebenso in der vorläufigen Modellierung ( $3 \%$ aller auftretenden MID). Ein Grund könnte sein, dass mündliche Aushandlungen der schriftlichen Fixierung vorgelagert sind.

\subsection{Modalitätsinterdependenzen als funktionale Verdichtungsstellen}

Betrachtet man MID-Funktionen interaktionstypübergreifend, so fällt auf, dass Modalitätsinterdependenzen häufig Kristallisationspunkte mehrerer Handlungen und Aufgaben sind: Mehrere Aufgaben werden synergetisch bearbeitet, so dass Modalitätsinterdependenzen größtenteils multifunktional sind (z. B. Handlungsschema 2: die Aufgabe Informationsrekonstruktion kombiniert mit der Aufgabe Prozessmodellierung und der Handlung Prozesselemente schriftlich notieren). ${ }^{8}$ In Anlehnung an Kallmeyer (1995: 9) könnte man auch von MID als funktionale Verdichtungsstellen sprechen. ${ }^{2}$ In diesem Punkt zeigt sich die Leistungsfähigkeit eines multimodalen Ausdrucks- und Handlungsspektrums in der professionellen Interaktion. In den stark kooperativ ausgerichteten Interaktionstypen 1 (92\% aller auftretender MID sind multifunktional) und 2 (77\% multifunktionale MID) dominiert Multifunktionalität von MID, monofunktionale MID sind selten (vgl. Tab. 2 im Anhang). In Interaktionstyp 3 ist das Verhältnis von mono- und multifunktionalen MID umgekehrt (70\% monofunktionale MID zu 30\% multifunktionalen MID).

\section{$4 \quad$ Fazit und Ausblick}

Modalitätsinterdependenzen sind im Fallbeispiel funktional relevant in Phasen der Generierung und Transformation von Wissen zum erhobenen Produktionsprozess. Die kombinierte Nutzung von Sprechen, Schreiben und/oder Visualisieren ist das Mittel der Wahl, aufgabenreiche und komplexe Anforderungen im professionellen Kontext kollaborativ und effizient zu bewältigen. Zum spezifischen instrumentellen Beitrag, den MID bei der Aufgabenerfüllung leisten, sind drei Punkte festzuhalten:

- Funktionen von MID liegen in zwei großen Handlungsbereichen kooperativer Zusammenarbeit:

1. Erheben und Beschreiben von Wissen durch Verdauerung und Materialisieren zur Informationsbereitstellung für nachfolgende Interaktionen (in Interaktionstyp 1 und 2). Hier

\footnotetext{
8 Multifunktional erfasst MID-Fälle, bei denen eine oder mehrere Aufgaben durch mehrere Handlungen der Interaktionsbeteiligten bearbeitet werden. Monofunktional erfasst Fälle von MID, bei denen eine Aufgabe durch eine Handlung bearbeitet wird.

${ }^{9}$ Kallmeyer (1995: 9) verwendet diesen Ausdruck im Zusammenhang mit typischen Aktivitäten zur Bildung eines sozialen Stils und spricht davon, ,wie die Beteiligten die Bündelung verschiedener Ausdrucksmittel als motivierte Wahl durchführen“.
} 
erfüllen Schreiben und Visualisieren in Kombination mit Sprechen objektivierende, validierende, aber auch epistemische Funktionen im Sinne eines epistemischen Schreibens (vgl. Raible 2004: 202).

2. Klären, Vermitteln und Revidieren von Wissen (in Interaktionstyp 2 und 3). Hier ist die interaktive Aushandlung und Festsetzung von Wissensbestandteilen für ein finales Produkt (K3-Prozessmodell) entscheidend.

Die Nutzung mehrerer Modalitäten im Zusammenspiel als Modalitätsinterdependenzen ist für die Operationalisierung und Optimierung von Prozessabläufen im Kontext einer industriellen Prozessmodellierung zentral. Hier zeigt sich auch die Wichtigkeit, MID systematisch zu untersuchen, etwa mithilfe des im Beitrag vorgeschlagenen Modells zu Formen und Funktionen von MID.

- MID dienen entweder der Bearbeitung einer Aufgabe oder mehrere Aufgaben fallen zusammen, was funktional und erwartbar ist. MID sind funktionale Verdichtungsstellen im Interaktionsgeschehen, bei denen in der Hauptsache mehrere Aufgaben multifunktional umgesetzt werden.

- MID sind handlungsschemaimmanent funktional. Es gibt nur zwei Aufgaben, die scheinbar keiner MID bedürfen (Handlungsschema 1: Interaktionseröffnung und -beendigung). Die Fokussierung und Orientierung auf den Interaktionsbeteiligten bedingt in der Gesprächssituation Verbalität. In diesem Kontext wäre in weiteren Studien interessant zu klären, welche Handlungsschema, Aufgaben und Aufgabentypen eine hohe Affinität zu MID haben und welche Aufgaben und Handlungen ,resistent' gegenüber MID sind und warum dies so ist.

Weiterhin zu untersuchen ist die Rolle handschriftlicher Notizen als Objekte, die interaktionsbegleitend in allen drei Gesprächssituationen des Fallbeispiels entstehen. Die Notizen bauen in Teilen aufeinander auf, verweisen inhaltlich aufeinander und werden situationsübergreifend, mal mehr mal weniger gemeinsam, als Arbeitsmittel genutzt. Sie dienen als intermediäre Objekte, d. h. als kooperativ genutzte Arbeitsobjekte, die Zwischenergebnisse festhalten und für weitere Bearbeitungsphasen anschlussfähig sind (vgl. Vinck 1999, Pitsch 2007: 64). Intermediäre Objekte machen als materielle (schriftliche und grafisch-symbolische) Spuren Prozesse der Wissenskonstruktion und -weitergabe in professionellen Interaktionen sichtbar. Dabei sind Schreiben und Visualisieren ,epistemische Verfahren [...], die im Akt der Aufzeichnung an der Entfaltung von Gegenständen des Wissens teilhaben“ (Hoffmann 2008: 7). In diesem Zusammenhang wären die Notizen prozess- und produktbezogen, in ihrer zunehmenden Kondensierung im Verlauf der Prozessmodellierung sowie in ihren Funktionen zu analysieren, insbesondere hinsichtlich ihrer vielfältigen grafisch-symbolischen Anteile (vgl. Ullrich/Jakobs 2019/in Vorb.).

\section{Literaturverzeichnis}

Allweyer, Thomas (2009): Geschäftsprozessmanagement. Strategie, Entwurf, Implementierung, Controlling. Herdecke/Witten: W3L-Verlag.

Best, Eva/Weth, Martin (2007): Geschäftsprozesse optimieren. Der Praxisleitfaden für erfolgreiche Reorganisation. 2. überarbeitete Auflage. Wiesbaden: Gabler. 
Brünner, Gisela (2000): Wirtschaftskommunikation. Linguistische Analyse ihrer mündlichen Formen. Tübingen: Niemeyer.

Bucher, Hans-Jürgen (2011): „Multimodales Verstehen oder Rezeption als Interaktion. Theoretische und empirische Grundlagen einer systematischen Analyse der Multimodalität". In: Diekmannshenke, Hajo/Klemm, Michael/Stöckl, Hartmut (eds.): Bildlinguistik. Theorie Methoden - Fallbeispiele. Berlin, Erich Schmidt: 123-156.

Fiehler, Reinhard/Kindt, Walther/Schnieders, Guido (2002): „Kommunikationsprobleme in Reklamationsgesprächen“. In: Brünner, Gisela/Fiehler, Reinhard/Kindt, Walther (eds.): Angewandte Diskursforschung. Radolfzell, Verlag für Gesprächsforschung: 120-154.

Fiehler, Reinhard (2009): „Gesprochene Sprache“. In: Duden. Die Grammatik. 8. überarbeitete Auflage. Hg. von der Dudenredaktion ( = Duden 4). Mannheim/Wien/Zürich, Dudenverlag: $1165-1244$.

Fricke, Ellen (2012): Grammatik multimodal. Wie Wörter und Gesten zusammenwirken. Berlin/Boston: de Gruyter.

Habscheid, Stephan et al. (2015): „Sprache in Organisationen“. In: Felder, Ekkehard/Gardt, Andreas (ed.): Handbuch Sprache und Wissen. Berlin/Boston, de Gruyter: 392-410.

Hausendorf, Heiko (2015): „Interaktionslinguistik“. In: Eichinger, Ludwig M. (ed.): Sprachwissenschaft im Fokus. Positionsbestimmungen und Perspektiven. Berlin/München/Boston, de Gruyter: 43-70.

Hoffmann, Christoph (2008): „Festhalten, Bereitstellen. Verfahren der Aufzeichnung“. In: Ders. (ed.): Daten sichern. Schreiben und Zeichnen als Verfahren der Aufzeichnung. Zürich/Berlin, Diaphanes: 7-20.

Jakobs, Eva-Maria (2011): „Multimodale Fachkommunikation“. In: Baumann, Klaus-Dieter (ed.): Fach - Translat - Kultur. Interdisziplinäre Aspekte der vernetzten Vielfalt. Berlin, Frank \& Timme: 106-124.

Jakobs, Eva-Maria et al. (2011): „Industrielle Prozessmodellierung als kommunikativer Prozess. Eine Typologie zentraler Probleme“. Gesprächsforschung - Online-Zeitschrift zur verbalen Interaktion 12: 223-264. www.gespraechsforschung-online.de/heft2011/ heft2011.html [06.02.2019].

Kallmeyer, Werner (1985): „Handlungskonstitution im Gespräch. Dupont und sein Experte führen ein Beratungsgespräch“. In: Gülich, Elisabth/Kotschi, Thomas (eds.): Grammatik, Konversation, Interaktion. Tübingen, Niemeyer: 81-122.

Kallmeyer, Werner (1995): „Zur Darstellung von kommunikativem sozialem Stil in soziolinguistischen Gruppenporträts“. In: Keim, Inken (ed.): Kommunikative Stilistik einer sozialen Welt „kleiner Leute“ in der Mannheimer Innenstadt. Berlin/New York, de Gruyter: 1-25.

Kallmeyer, Werner/Schütze, Fritz (1977): „Zur Konstitution von Kommunikationsschemata der Sachverhaltsdarstellung“. In: Wegner, Dirk (ed.). Gesprächsanalyse. Hamburg, Buske: 159-274.

Klug, Nina-Maria/Stöckl, Hartmut (2015): „Sprache im multimodalen Kontext“. In: Felder, Ekkehard/Gardt, Andreas (ed.): Handbuch Sprache und Wissen. Berlin/Boston, de Gruyter: 242-264.

Lehnen, Katrin (2017): „Kooperatives Schreiben“. In: Becker-Mrotzek, Michael/Grabowski, Joachim/Steinhoff, Torsten (eds.): Forschungshandbuch empirische Schreibdidaktik. Münster, Waxmann: 299-314. 
Lehnen, Katrin/Gülich, Elisabeth (1997): „Mündliche Verfahren der Verschriftlichung: Zur interaktiven Erarbeitung schriftlicher Formulierungen“. Zeitschrift für Literaturwissenschaft und Linguistik 27/108: 108-136.

Mittelberg, Irene/Schmitz, Thomas H./Groninger, Hannah (2017): „Operative Manufakte. Gesten als unmittelbare Skizzen in frühen Stadien des Entwurfsprozesses“. In: Ammon, Sabine/Hinterwaldner, Inge (eds.): Bildlichkeit im Zeitalter der Modellierung. Operative Artefakte in Entwurfsprozessen der Architektur und des Ingenieurwesens. Paderborn, Fink: 5786.

Mondada, Lorenza/Svinhufvud, Kimmo (2016): „Writing-in-interaction. Studying writing as a multimodal phenomenon in social interaction“. Language and Dialogue 6/1: 1-53.

Müller, Cornelia (2010): „Wie Gesten bedeuten. Eine kognitiv-linguistische und sequenzanalytische Perspektive“. Sprache und Literatur 41: 37-68.

Müller, Andreas P. (2006): Sprache und Arbeit. Aspekte einer Ethnographie der Unternehmenskommunikation. Tübingen: Narr.

Pitsch, Karola (2007): „Unterrichtskommunikation revisited. Tafelskizzen als interaktionale Ressource“. Bulletin Suisse de Linguistique Appliquée. Special Issue: Die Sprache betrachten. Videodaten in der sprachwissenschaftlichen Forschung: 59-80.

Pitsch, Karola/Krafft, Ulrich (2010): „Von der emergenten Erfindung zu konventionalisiert darstellbarem Wissen. Zur multimodalen Entwicklung gemeinsamer räumlicher Vorstellungen bei Designern“. In: Dausendschön-Gay, Ulrich/Domke, Christine/Ohlhus, Sören (eds.): Wissen in (Inter-)Aktion. Verfahren der Wissensgenerierung in unterschiedlichen Praxisfeldern. Berlin/New York, de Gruyter: 189-221.

Raible, Wolfgang (2004): „Über das Entstehen der Gedanken beim Schreiben“. In: Krämer, Sybille (ed.): Performativität und Medialität. München, Fink: 191-214.

Reimer, Eva et al. (2015): „Annotating Modality Interdependencies“. Proceedings of the Int. Conference of the German Society of Computational Linguistics and Language Technology: 110-111.

Reimer, Eva et al. (2017): „Mehrebenenannotation multimodaler Daten“. Gesprächsforschung - Online-Zeitschrift zur verbalen Interaktion 18: 91-116. www.gespraechsforschung-online.de/2017.html [06.02.2019].

Schmidt, Thomas/Wörner, Kai (2014): „EXMARaLDA“. In: Durand, Jacques/Gut, U1rike/Kristoffersen, Gjert (ed.): The Oxford Handbook of Corpus Phonology. Oxford, OUP: 402-419.

Schmitt, Reinhold (2005): „Zur multimodalen Struktur von turn-taking“. Gesprächsforschung - Online-Zeitschrift zur verbalen Interaktion 6: 17-61.

Schmitt, Reinhold (2013): Körperlich-räumliche Aspekte der Interaktion. Tübingen: Narr.

Spiegel, Carmen/Spranz-Fogasy (2001): „Zur Methodologie der Handlungsstrukturanalyse von Gesprächen“. In: Iványi, Zsuzsanna/Kertész, András (eds.): Gesprächsforschung. Tendenzen und Perspektiven. Frankfurt a.M. etc., Lang: 243-257.

Spranz-Fogasy, Thomas (1997): Interaktionsprofile. Die Herausbildung individueller Handlungstypik in Gesprächen. Opladen: Westdeutscher Verlag.

Stöckl, Hartmut (2006): „Zeichen, Text und Sinn - Theorie und Praxis der multimodalen Textanalyse“. In: Eckkrammer, Eva Martha/Held, Gudrun (eds.): Textsemiotik. Studien zu multimodalen Texten. Frankfurt a. M., Lang: 11-36. 
Trevisan, Bianka et al. (2016): „Digital Humanities und Linguistik: Herausforderungen und ihre Potentiale am Beispiel der Annotation multimodaler Daten“. In: DHd 2016: Modellierung, Vernetzung, Visualisierung. Die Digital Humanities als fächerübergreifendes Paradigma. Konferenzabstracts. Duisburg, nisaba: 382-385.

Ullrich, Anna Valentine/Jakobs, Eva-Maria/Eraßme, Denise (2016): „'ich schreib das mal hier rein ähm'. Modality-taking - Schreibhinweise in professionellen mündlichen Interaktionssituationen“. Glottotheory 7/1: 67-88.

Ullrich, Anna Valentine/Reimer, Eva/Jakobs, Eva-Maria (2017): „Modalitätsinterdependenzen im professionellen Kontext: Formen und Indikatoren“. ZfAL 67: 51-79.

Ullrich, Anna Valentine/Jakobs, Eva-Maria (2019): Zeichnen in mündlichen Arbeitssituationen [in Vorbereitung].

Vinck, Dominique (1999): „Les objets intermédiaires dans les réseaux de cooperation scientifique. Contribution à la prise en compte des objets dans les dynamiques sociales“. Revue française de sociologie 40/2: 385-414.

\section{Anhang}

\begin{tabular}{|l||c|c|c|c|c|c|}
\hline & Anzahl & \multirow{2}{*}{$\begin{array}{l}\text { Anzahl Interak-ti- } \\
\text { Token }\end{array}$} & \multicolumn{5}{|c|}{ Datensätze je Interaktionstyp } \\
\cline { 3 - 7 } & onsketten $^{10}$ & 1 & 2 & 3 & $\Sigma$ \\
\hline Pilotkorpus & 9.075 & 1 & 1 & 1 & 2 & 4 \\
\hline Goldstandard & 33.185 & 5 & 5 & 5 & 9 & 19 \\
\hline Gesamtkorpus & 70.950 & 10 & 11 & 11 & 33 & 55 \\
\hline
\end{tabular}

Tabelle 1: Datenbasis zum Fallbeispiel einer industriellen Prozessmodellierung

\begin{tabular}{|c|c|c|c|}
\hline Modalitätsinterdependenzen & $\begin{array}{l}\text { Interaktionstyp } \\
1\end{array}$ & $\begin{array}{l}\text { Interaktionstyp } \\
2\end{array}$ & $\begin{array}{l}\text { Interaktionstyp } \\
3\end{array}$ \\
\hline MID interpersonell & 59 & 119 & 8 \\
\hline MID intrapersonell & 29 & 26 & 49 \\
\hline MID Sprechen-Schreiben interpersonell & 54 & 115 & 2 \\
\hline MID Sprechen-Schreiben intrapersonell & 22 & 22 & 3 \\
\hline MID Sprechen-Visualisieren interpersonell & 0 & 1 & 4 \\
\hline MID Sprechen-Visualisieren intrapersonell & 3 & 3 & 33 \\
\hline $\begin{array}{l}\text { MID Sprechen-Schreiben-Visualisieren } \\
\text { interpersonell }\end{array}$ & 5 & 3 & 2 \\
\hline $\begin{array}{l}\text { MID Sprechen-Schreiben-Visualisieren } \\
\text { intrapersonell }\end{array}$ & 4 & 1 & 13 \\
\hline MID multifunktional & 81 & 111 & 17 \\
\hline MID monofunktional & 7 & 34 & 40 \\
\hline MID insgesamt & 88 & 145 & 57 \\
\hline
\end{tabular}

Tabelle 2: Ergebnisse zu Modalitätsinterdependenzen im Fallbeispiel der industriellen Prozess-modellierung (Goldstandard)

\footnotetext{
10 Als Interaktionsketten wurden Datensätze zu Interaktionen zusammengestellt, die sich inhaltlich aufeinander beziehen und in der Zeit nacheinander in Interaktionstyp 1, 2 und 3 auftreten (vgl. Reimer et al. 2017: 98).
} 